\title{
Secretory-Stage Ameloblast
}

National Cancer Institute

\section{Source}

National Cancer Institute. Secretory-Stage Ameloblast. NCI Thesaurus. Code C33524.

A cylindrical cell in the innermost layer of the enamel organ which deposits the organic matrix of enamel to create tooth enamel on the surface of the developing tooth. 